\title{
Correlation and Path Coefficient Analysis of Single Cross Hybrids in Maize (Zea mays L.)
}

\author{
Saripalli Varalakshmi*, Mruthunjaya C. Wali, S.K. Deshpande and S.I. Harlapur \\ Department of Genetics and Plant Breeding, University of agricultural sciences, \\ Dharwad-580005, Karnataka, India \\ *Corresponding author
}

\section{A B S T R A C T}

Keywords

Maize, Correlation, Path analysis, Grain yield

Article Info

Accepted:

16 March 2018

Available Online:

10 April 2018
The yield is a complex trait, which is highly influenced by the environment and hence indirect selection through component traits would be an advisable strategy to increase the efficiency of selection. Fifty two F1's generated by crossing 26 lines with 2 testers in line $\times$ tester fashion were evaluated in randomized block design along with three standard checks with two replications. Plant height, ear height, cob length, cob girth, number of rows per ear and test weight recorded significant positive correlation both at genotypic and phenotypic level. However, path analysis revealed that days to $50 \%$ tasseling, number of rows per ear and test weight revealed high positive direct effect on grain yield.

\section{Introduction}

Maize (Zea mays L.) is one of the most superior cereal crops in the world due to its wide adaptation and varieties of utilisation. The expansion of maize to new areas and environment still continues, as it has wide range of plasticity and hence the suitability of maize to diverse environments is unmatched by any other crop. It plays a significant role in human and livestock nutrition world-wide.

Grain yield in maize is a complex trait which is highly influenced by the external environment and the complexity is the result of complex interactions among different yield attributing traits. Hence, information regarding genotypic and phenotypic correlation coefficients among various plant traits help to ascertain the degree to which these traits are associated with economic productivity.

The association between two characters can directly be observed as phenotypic correlation while genotypic correlation expresses the extent to which two traits are genetically associated. Genotypic correlation results from linkage and pleotropy.

Path coefficient analysis measures the cause of association between two variables; it measures direct and indirect contribution of different 
yield attributing traits on yield. It is also equally beneficial as that of correlation studies since it is efficient biometrical tool which estimates direct contribution of each component trait and its effect through other component traits in influencing the yield. Hence present research was under taken to investigate correlation and path analysis in 52 F1's. Both genotypic and phenotypic correlations among and between pairs of yield traits provide scope for indirect selection in a crop breeding programme.

\section{Materials and Methods}

The present experiment was carried out at Main Agricultural Research Station (MARS), Dharwad. Base population was raised and crossing programme was conducted during rabi 2015-2016. The parents and $\mathrm{F}_{1} \mathrm{~s}$ developed were evaluated during kharif 2016. The experimental material for present study comprised of 26 newly received inbred lines from CIMMYT and two indigenous testers. The 52 single cross hybrids were developed using $\mathrm{L} \times \mathrm{T}$ mating design, by crossing 26 inbred lines with two testers during rabi 20152016.

The $52 \quad \mathrm{~F}_{1}$ hybrids generated during kharif 2016 were evaluated in RBD (Randomized Block Design) with two replications along with parental lines and three commercial checks (GH0727, BIO-9681 and 900 M Gold). Each entry was raised in two rows of four meter length following a spacing of $60 \mathrm{x}$ $20 \mathrm{~cm}$.

The observations on twelve quantitative characters namely plant height $(\mathrm{cm})$, ear height $(\mathrm{cm})$, days to 50 per cent tasseling, days to 50 per cent silking, cob length $(\mathrm{cm})$, cob girth $(\mathrm{cm})$, number of kernel rows per ear, number of kernels per row, test weight $(\mathrm{g})$, Shelling percentage, brown husk maturity and grain yield (q/ha) were recorded. Observations related to days to 50 percent tasseling, days to 50 percent silking, brown husk maturity and grain yield (q/ha) were recorded on plot basis while other characters were recorded on five randomly selected competitive plants leaving border plants of each row.

\section{Results and Discussion}

The results indicated that genotypic correlation was slightly higher than phenotypic correlation for most of the characters, suggesting that there was a strong inherent association among various characters studied and they were governed by additive gene action. The genotypic and phenotypic correlation coefficients among yield and yield attributing traits are presented in Table 1. Grain yield had highly significant and positive correlation with cob girth, number of kernel rows per ear, test weight and plant height. Similar results were found by Majidkhayatnezhad et al., (2010) and Pavan et al., (2011). The traits showing significant positive correlation with grain yield can be improved simultaneously in a selection programme, because of mutual relationships that exist among traits.

Sometimes, correlation coefficients give misleading results because the correlation between two variables may be due to third factor. It is therefore necessary to analyse the cause and effect relationship between dependent and independent variables to entangle the nature of relationship between the variables.

Path coefficient analysis (Dewey and $\mathrm{Lu}$, 1957) furnished a method partitioning the correlation coefficient into direct and indirect effect and provides the information on actual contribution of a trait on the yield.

The results of phenotypic and genotypic path coefficient analysis are presented in Table 2. 
Table.1 Phenotypic and genotypic correlation coefficient for grain yield and its components in maize

\begin{tabular}{|c|c|c|c|c|c|c|c|c|c|c|c|c|c|}
\hline & & $\mathrm{X} 1$ & $\mathrm{X} 2$ & $\mathrm{X} 3$ & $\mathrm{X} 4$ & $\mathrm{X} 5$ & $\mathrm{X} 6$ & $\mathrm{X} 7$ & $\mathrm{X} 8$ & X9 & $\mathrm{X} 10$ & X11 & GY(g/ha) \\
\hline \multirow[t]{2}{*}{$\mathrm{X} 1$} & $\mathrm{P}$ & 1.000 & $0.645^{* *}$ & 0.108 & 0.134 & $0.243 * *$ & $0.173^{*}$ & $0.183^{*}$ & 0.065 & 0.143 & 0.038 & 0.102 & $0.313 * *$ \\
\hline & G & 1.000 & $0.610^{* *}$ & $0.165^{*}$ & $0.211^{* *}$ & $0.320 * *$ & $0.484 * *$ & 0.449 ** & 0.061 & $0.323 * *$ & -0.002 & $0.185^{*}$ & $0.427 * *$ \\
\hline \multirow[t]{2}{*}{$\mathrm{X} 2$} & $\mathrm{P}$ & & 1.000 & -0.092 & -0.092 & -0.098 & 0.005 & 0.115 & -0.119 & 0.119 & 0.098 & -0.050 & $0.157^{*}$ \\
\hline & G & & 1.000 & -0.122 & -0.106 & $-0.214 * *$ & 0.090 & $0.168^{*}$ & $-0.344 * *$ & $0.252 * *$ & 0.105 & -0.085 & $0.188^{*}$ \\
\hline \multirow[t]{2}{*}{$\mathbf{X 3}$} & $\mathrm{P}$ & & & 1.000 & $0.955^{* *}$ & $0.680 * *$ & $0.304 * *$ & -0.090 & $0.174 *$ & $0.300^{* *}$ & $-0.193 *$ & 0.032 & 0.124 \\
\hline & G & & & 1.000 & $0.986^{* *}$ & $0.799 * *$ & $0.434 * *$ & -0.152 & $0.251^{* *}$ & 0.380 ** & $-0.234 * *$ & 0.030 & $0.154^{*}$ \\
\hline \multirow[t]{2}{*}{$\mathrm{X} 4$} & $\mathrm{P}$ & & & & 1.000 & $0.692 * *$ & $0.338^{* *}$ & -0.066 & $0.197 *$ & $0.308^{* *}$ & $-0.188^{*}$ & 0.050 & 0.095 \\
\hline & G & & & & 1.000 & $0.859 * *$ & $0.611^{* *}$ & -0.065 & $0.257 * *$ & $0.443 * *$ & $-0.222 * *$ & 0.097 & 0.123 \\
\hline \multirow[t]{2}{*}{$\mathrm{X5}$} & $\mathrm{P}$ & & & & & 1.000 & $0.483^{* *}$ & 0.109 & $0.154 *$ & $0.435 * *$ & 0.042 & $0.353^{* *}$ & $0.245 * *$ \\
\hline & G & & & & & 1.000 & $0.639 * *$ & -0.020 & $0.302 * *$ & $0.493 * *$ & 0.015 & $0.344^{* *}$ & $0.291 * *$ \\
\hline \multirow[t]{2}{*}{$\mathrm{X6}$} & $\mathrm{P}$ & & & & & & 1.000 & $0.351 * *$ & $0.287 * *$ & $0.766^{* * *}$ & -0.010 & $0.436^{* *}$ & $0.172 *$ \\
\hline & G & & & & & & 1.000 & $0.198^{*}$ & $0.487 * *$ & $0.813^{* *}$ & $0.207^{* *}$ & $0.448^{* *}$ & $0.244 * *$ \\
\hline \multirow[t]{2}{*}{$\mathbf{X 7}$} & $\mathrm{P}$ & & & & & & & 1.000 & $0.392 * *$ & 0.144 & -0.066 & $0.689^{* *}$ & $0.365 * *$ \\
\hline & G & & & & & & & 1.000 & $0.430^{* * *}$ & -0.111 & -0.074 & $0.783^{* *}$ & $0.542 * *$ \\
\hline \multirow[t]{2}{*}{$\mathrm{X8}$} & $\mathrm{P}$ & & & & & & & & 1.000 & 0.1427 & -0.077 & 0.034 & $0.313^{* *}$ \\
\hline & G & & & & & & & & 1.000 & $0.158^{*}$ & -0.101 & 0.081 & $0.489 * *$ \\
\hline \multirow[t]{2}{*}{$\mathbf{X 9}$} & $\mathrm{P}$ & & & & & & & & & 1.000 & 0.151 & 0.141 & 0.103 \\
\hline & G & & & & & & & & & 1.000 & $0.299 * *$ & 0.048 & $0.163^{*}$ \\
\hline \multirow[t]{2}{*}{$\mathrm{S} \%$} & $\mathrm{P}$ & & & & & & & & & & 1.000 & 0.018 & 0.137 \\
\hline & G & & & & & & & & & & 1.000 & 0.087 & 0.148 \\
\hline \multirow[t]{2}{*}{ TW } & $\mathrm{P}$ & & & & & & & & & & & 1.000 & $0.308 * *$ \\
\hline & G & & & & & & & & & & & 1.000 & $0.386^{* *}$ \\
\hline
\end{tabular}

Significance Levels $0.05 \%$; 0.01\%, If correlation $r=>0.1524 ; 0.1994$

$\mathrm{X} 1$ - Plant height $(\mathrm{cm}) \quad \mathrm{X} 5-$ Brown husk maturity

$\mathrm{X} 2$ - Ear height $(\mathrm{cm})$

X6 - Cob length $(\mathrm{cm})$

$\mathrm{X} 7-\mathrm{Cob}$ girth $(\mathrm{cm})$

$\mathrm{X} 8$ - Number of kernel rows per ear

$\mathrm{X} 4$ - Days to $50 \%$ silking
X9 - Number of kernels per row

X10 - Shelling percentage

X11 - 100 grain weight $(\mathrm{g})$

Table.2 Phenotypic and genotypic path coefficient for grain yield and its components in maize



\begin{tabular}{|c|c|c|c|c|}
\hline & $\mathbf{X 1}$ & $\mathbf{X} 2$ & $\mathbf{X 3}$ & $\mathbf{X} 4$ \\
\hline P & $\mathbf{0 . 2 2 7}$ & 0.146 & 0.024 & 0.030 \\
\hline G & $\mathbf{0 . 1 2 6}$ & 0.363 & 0.363 & -0.546 \\
\hline P & 0.027 & $\mathbf{0 . 0 4 3}$ & -0.004 & -0.004 \\
\hline G & 0.077 & $\mathbf{0 . 5 9 6}$ & -0.267 & 0.275 \\
\hline P & 0.057 & -0.048 & $\mathbf{0 . 5 3 0}$ & 0.507 \\
\hline G & 0.021 & -0.072 & $\mathbf{2 . 1 9 2}$ & -2.544 \\
\hline P & -0.065 & 0.045 & -0.466 & $\mathbf{- 0 . 4 8 8}$ \\
\hline G & 0.026 & -0.063 & 2.162 & $\mathbf{- 2 . 5 7 9}$ \\
\hline P & 0.020 & -0.008 & 0.055 & 0.056 \\
\hline G & 0.040 & -0.127 & 1.752 & -2.217 \\
\hline P & -0.024 & -0.0008 & -0.043 & -0.047 \\
\hline G & 0.061 & 0.053 & 0.952 & -1.577 \\
\hline P & 0.017 & 0.010 & -0.008 & -0.006 \\
\hline G & 0.057 & 0.100 & -0.335 & 0.169 \\
\hline P & 0.019 & -0.035 & 0.052 & 0.059 \\
\hline G & 0.007 & -0.205 & 0.552 & -0.665 \\
\hline P & 0.002 & 0.001 & 0.004 & 0.005 \\
\hline G & 0.041 & 0.150 & 0.833 & -1.144 \\
\hline P & 0.005 & 0.015 & $-0,029$ & -0.028 \\
\hline G & -0.0003 & 0.062 & -0.514 & 0.573 \\
\hline P & 0.025 & -0.012 & 0.007 & 0.012 \\
\hline G & 0.023 & -0.051 & 0.065 & -0.250 \\
\hline
\end{tabular}

$\mathrm{X} 1$ - Plant height $(\mathrm{cm})$

$\mathrm{X} 2$ - Ear height $(\mathrm{cm})$

X3 - Days to $50 \%$ tasselling

$\mathrm{X} 4$ - Days to $50 \%$ silking

\begin{tabular}{|c|c|}
\hline $\mathbf{X 5}$ & $\mathbf{X 6}$ \\
\hline & \\
\hline 0.055 & 0.039 \\
\hline 0.166 & -0.115 \\
\hline-0.004 & 0.0002 \\
\hline 0.111 & -0.021 \\
\hline 0.360 & 0.161 \\
\hline 0.415 & -0.103 \\
\hline-0.337 & -0.165 \\
\hline 0.446 & -0.145 \\
\hline $\mathbf{0 . 0 8 2}$ & 0.039 \\
\hline $\mathbf{0 . 5 1 9}$ & -0.152 \\
\hline-0.068 & $\mathbf{- 0 . 1 4 1}$ \\
\hline 0.332 & $\mathbf{- 0 . 2 3 7}$ \\
\hline 0.010 & 0.033 \\
\hline-0.010 & -0.047 \\
\hline 0.046 & 0.086 \\
\hline 0.157 & -0.116 \\
\hline 0.007 & 0.012 \\
\hline 0.256 & -0.193 \\
\hline 0.006 & -0.001 \\
\hline 0.008 & -0.049 \\
\hline 0.087 & 0.107 \\
\hline 0.178 & -0.106 \\
\hline
\end{tabular}

\begin{tabular}{|c|}
\hline $\mathbf{X 7}$ \\
\hline 0.041 \\
\hline-0.102 \\
\hline 0.005 \\
\hline-0.038 \\
\hline-0.047 \\
\hline 0.034 \\
\hline 0.032 \\
\hline 0.014 \\
\hline 0.009 \\
\hline 0.004 \\
\hline-0.049 \\
\hline-0.045 \\
\hline $\mathbf{0 . 0 9 4}$ \\
\hline $\mathbf{- 0 . 2 2 7}$ \\
\hline 0.117 \\
\hline-0.097 \\
\hline 0.002 \\
\hline 0.025 \\
\hline-0.010 \\
\hline 0.016 \\
\hline 0.170 \\
\hline-0.177 \\
\hline
\end{tabular}
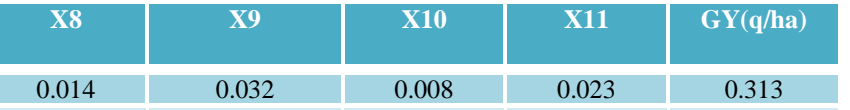

\subsection{9}

\begin{tabular}{l|l}
-0.005 & 0.005
\end{tabular}

$-0.279$

0.092

0.204
-0.096

-0.096
0.209

0.012

0.245

$-0.040$

0.395

0.037

0.348

0.299

$\mathbf{0 . 8 1 0}$

0.002

0.128

$-0.011$

$-0.081$

0.008

0.005
0.003

$-0.0002$

0.004
0.007

0.159
0.005

$-0.102$

$-0.017$

$-0.150$

0.092
-0.016

0.035

0.003

$-0.108$

0.012

0.013

$-0.001$

0.042
0.002

0.002
$\mathbf{0 . 0 1 6}$

0.016
0.014

0.023

0.004

0.001

0.001

-0.006
-0.005

-0.005
-0.023
-0.007

-0.007
0.002

0.002
0.021
$\mathbf{0 . 1 5 3}$

$\mathbf{0 . 0 7 2}$

X5 - Brown husk maturity

X6 - Cob length $(\mathrm{cm})$

$\mathrm{X} 7$ - Cob girth $(\mathrm{cm})$

X9 - Number of kernels per row

$\mathrm{X} 10$ - Shelling percentage

X11 - 100 grain weight (g)

$\mathrm{X} 8$ - Number of kernel rows per ear 
Path coefficient analysis revealed that number of kernel rows per ear and test weight had maximum positive direct effect on grain yield indicating the effectiveness of direct selection for these traits, whereas direct and negative effects were exhibited by days to 50 per cent silking and cob length indicating that selection for these traits result in reduced grain yield. Similar results were observed by Hefny (2011), Nataraj et al., (2015) and Pavan et al., (2011).

The residual effect implies the role of other possible yield attributing traits which were not included in this study on grain yield. The residual effect recorded in this experiment is 0.4361 ; it indicates the characters used in our experiment explain around 60 per cent of variations which may be contributed to higher yields in maize. A similar result with respect to residual effect was observed by Begum et al., (2016).

\section{References}

Begum, S., Ahmed, A., Omy, S. H., Rohman, M. M. and Amiruzzaman, M., (2016). Genetic variability, character association and path analysis in maize (Zea mays L.). Bangladesh J. Agril. Res. 41(1): 173-182.
Dewey, D, R. and Lu, K. H., (1959). A correlation and pathcoefficient analysis of component of crested wheat grass seed production. Agron. J., 51(2): 515518.

Hefny, M., (2011). Genetic Parameters and Path Analysis of Yield and itsComponents in Corn Inbred Lines (Zea may L.) at Different Sowing Dates. Asian J. Crop Sci. 3(3):106-117.

Majid Khayatnezhad., Roza Gholamin., Shahzad Jamati-e-somarin and Rogayyeh Zabihi-e-eMahmoodabad. (2010). Correlation coefficient analysis between grain yield and its components in corn (Zea mays L.) hybrids. AmEuras. J. Agric. and Environ. Sci., 9(1): 105-108.

Nataraj, V., Shahi, J. P., Vandana, D., (2015). Character association and path analysis in maize (Zea may L.). Environment and Ecology, 33(1): 78-81.

Pavan, R., Lohithaswa, H. C., Wali, M. C., Prakash, G. and Shekara, B. G., (2011). Correlation and path coefficient analysis of grain yield and yield contributing traits in single cross hybrids of maize (Zea mays L.). Electron. J. Plant Br., 2(2):253-257.

\section{How to cite this article:}

Saripalli Varalakshmi, Mruthunjaya C. Wali, S.K. Deshpande and Harlapur, S.I. 2018. Correlation and Path Coefficient Analysis of Single Cross Hybrids in Maize (Zea mays L.). Int.J.Curr.Microbiol.App.Sci. 7(04): 1840-1843. doi: https://doi.org/10.20546/ijcmas.2018.704.209 